correlation coefficient (ICC) between predicted and real values. Pearson's $r$ was calculated too. The free and open source programming language $\mathrm{R}$ was used for all the analyses. Dataset source: Borráz-León, Javier I. (2020), "Dark triad, attractiveness, mate value, and sexual partners", Mendeley Data, V1, doi: 10.17632/87vx6jfnrp.1

Results: Machiavellianism, narcissism and psychopathy predictions obtained ICC of 0.593, 0.335, 0.505 and Pearson's $r$ of $0.608,0.346,0.548$ respectively. The results were indicative of fair performance, mainly for Machiavellianism and psychopathy.

Conclusions: AI might be useful to predict MNP. This could be utile in many situations, such as dealing with missing data or deciding whether to formally test someone. Finally, the AI used in this study is freely available, allowing anyone to experiment.

Disclosure: No significant relationships.

Keywords: Artificial Intelligence; Personality; traits; psychometry

\section{Posttraumatic stress disorder}

\section{O205}

The psychiatric impact of the 2020 beirut port explosion on civilians and relief workers

\section{R. Charara* and J. El-Khoury}

Psychiatry, American University of Beirut Medical Center, Beirut, Lebanon

${ }^{\star}$ Corresponding author.

doi: 10.1192/j.eurpsy.2021.387

Introduction: On August 4th 2020, a massive port explosion shook Beirut, killing at least 200, injuring more than 6,000 people and leaving more than a quarter of a million living in unfit homes. Various factors can participate in the severity of mental health outcomes of a disaster including the number of injuries, the degree of property destruction, unexpectedness of the occurrence of the event, and the type of the disaster.

Objectives: The main aim of this study is to assess the prevalence of post-traumatic stress disorder (acute stress disorder) and major depression at 1 and 6 months following the Beirut explosion. The secondary aim is to determine predictors of PTSD incidence among civilians and relief workers affected by the disaster.

Methods: This is a cross-sectional study with data collected via an online survey through convenience sampling. People will be recruited via social media platforms. To achieve a power of $80 \%$ and a two-sided significance of $5 \%$ and because gender differences will be explored, assuming a design effect (deff) of 2.5 , a minimum sample of 960 participants would be needed. The survey will include sociodemographic data, questions about exposure levels to trauma and a psychiatric symptom inventory. Pearson's Chi Square test will be used to examine the association between categorical variables and regression models will be run to examine the associations while controlling for confounders, including age, gender and others.

Results: The results from both rounds of data collection (months 1 and 6) will be available in late March 2021.

Conclusions: to follow based on results

Disclosure: No significant relationships.

Keywords: ptsd; community mental health; trauma

\section{6}

Post-traumatic stress, anxiety and depression after intensive care unit stay: Findings from a general hospital

\author{
U. Bhaumik $^{1 *}$, V. Subramaniyam ${ }^{2}$ and R. Kandukuru ${ }^{3}$ \\ ${ }^{1}$ Psychiatry, Independent Practice, Kolkata, India; ${ }^{2}$ Psychiatry, \\ Independent practice, Bangalore, India and ${ }^{3}$ Anaesthesiology And \\ Critical Care, M S Ramaiah Medical College, Bangalore, India \\ ${ }^{\star}$ Corresponding author.
}

doi: 10.1192/j.eurpsy.2021.388

Introduction: Post-traumatic stress disorder (PTSD) following intensive care is a relatively new entity. It is triggered due to traumatic experiences in a setting of threat to life due to illness. Prolonged stay in intensive care predisposes to delusional memories related to the stay experience and may increase likelihood of post-traumatic stress.It may also present as anxiety or depression. Objectives: This study explored the prevalence of post-traumatic symptoms in intensive care, find its correlates and its impact on health-related quality of life (HRQoL).

Methods: 225 adult patients admitted for at least 1 day in the intensive care unit (ICU) of a general hospital in Bangalore,India were recruited and assessed at 1 week, 1 month and 3 months after ICU discharge. Subjects were assessed for ICU related memories, PTSD, anxiety and depression scores and quality of life at and post discharge.

Results: $59.6 \%$ of the study population had significant posttraumatic stress, including anxiety in $62.35 \%$, depression in $10.58 \%$ and mixed anxiety-depression in $27.06 \%$. Delusional memories were found in $31.6 \%$. Presence of delusional memories was found to have significant correlation with post-traumatic stress and had a negative impact on HRQoL.

Conclusions: This study was the first of its kind from Asia. More systematic studies on PTSD following ICU stay and its correlates are required as available evidence lacks homogeneity. Suitable preventive measures should be taken to reduce prevalence of post-traumatic stress in intensive care due to its lasting impact on HRQoL.

Disclosure: No significant relationships.

Keywords: post-traumatic stress disorder; anxiety and depression; delusional memories; intensive care unit

\section{O207}

A comparison of mdma-assisted psychotherapy to non-assisted psychotherapy in treatment-resistant PTSD: A systematic review and meta-analysis

B. Illingworth ${ }^{1 \star}$, D. Lewis ${ }^{2}$, A. Lambarth ${ }^{3}$, K. Stocking ${ }^{4}$, J. Duffy ${ }^{5}$, L. Jelen ${ }^{6}$ and J. Rucker ${ }^{6}$

${ }^{1}$ Respiratory Medicine, Cambridge University Hospitals NHS Foundation Trust, Cambridge, United Kingdom; ${ }^{2}$ Acute Medicine, Brighton \& Sussex University Hospitals NHS Trust, Brighton, United Kingdom; ${ }^{3}$ Clinical Pharmacology, University College London

Hospitals NHS Foundation Trust, London, United Kingdom; ${ }^{4}$ Centre For Biostatistics, University of Manchester, Manchester, United Kingdom; ${ }^{5}$ Institute For Women's Health, University College London, London, United Kingdom and ${ }^{6}$ Centre For Affective Disorders, Institute of Psychiatry, Psychology \& Neuroscience, King's College London, London, United Kingdom

${ }^{*}$ Corresponding author.

doi: 10.1192/j.eurpsy.2021.389 